\title{
Diseño de un modelo de evaluación y desarrollo docente en una universidad privada
}

\author{
Teresa de Dios Alija ${ }^{1}$; José Manuel García Ramos ${ }^{2}$; Soraya Muñoz Pérez ${ }^{3}$
}

Recibido: marzo 2015 / Evaluado: junio 2015 / Aceptado: julio 2015

Resumen. La universidad que quiera responder al principio de excelencia académica, debe conocer de manera exhaustiva el desarrollo e impacto de la actividad docente, investigadora y de gestión de los profesores que en ella desempeñan su labor profesional. Para ello es necesario establecer un procedimiento riguroso que proporcione información válida y suficiente acerca de los procesos que contribuyen a lograr los objetivos estratégicos de la universidad, los medios que propician la mejora continua de los protocolos de enseñanza-aprendizaje, el modo en que se implican los responsables en el ejercicio del liderazgo en la organización, el compromiso de los profesores, los métodos de identificación de necesidades de formación y desarrollo y las maneras en que se incentiva la calidad esperada y percibida y la excelencia docente (reconocimiento). De esta manera podrán tomarse decisiones convenientes y oportunas para mejorar los aspectos críticos detectados y potenciar aquellos cuya valoración ha sido positiva.

Para validar una propuesta de este tipo, en la Universidad Francisco de Vitoria (en adelante UFV) se ha implementado un sistema de desarrollo y evaluación del desempeño docente que tiene la pretensión de superar algunas de las limitaciones del Programa DOCENTIA, adecuando el desarrollo de las competencias profesionales de los profesores a las expectativas organizacionales expresadas en las líneas estratégicas de la universidad. Para ello, define las competencias, acciones y comportamientos profesionales y personales relevantes para el cumplimiento de la misión universitaria, integrando las misiones y objetivos en un sistema de mejora profesional y personal acompañado por el director académico, recompensando la excelencia en términos estratégicos y organizativos, y permitiendo que los sistemas de delegación, de autonomía y de dirección de los profesores se ajusten, se redefinan y se adapten al sistema de gobierno de la universidad.

Palabras clave: evaluación del desempeño docente; calidad docente; competencias del profesor universitario; dirección por misiones.

\section{[en] Design of a model of teacher evaluation and development in a private university}

Abstract. The university that wants to respond to the principle of academic excellence must thoroughly know the development and impact of teaching, research and management activity of the

1 Universidad Francisco de Vitoria (España)

E-mail: t.dedios.prof@ufv.es

2 Universidad Complutense de Madrid (España)

E-mail: jmgramos@ucm.es

3 Universidad Francisco de Vitoria (España)

E-mail: s.munoz@ufv.es 
teachers who perform their work in the academic institution. This requires the establishing of a rigorous process that provides valid and sufficient information about the processes that contribute to achieve the university's strategic objectives, the means that propitiate the continuous improvement of the teaching-learning protocols, the way in which leaders get involve in the organization, the commitment of teachers, the methods for identifying training and development needs, and the ways the expected and perceived quality are encouraged for each stakeholder (acknowledgement). In this way appropriate and timely decisions can be made to improve the critical aspects detected and to enhance those whose assessment has been positive.

To validate a proposal of this kind, the Francisco de Vitoria University has implemented a system for the development and evaluation of teaching performance that aims to overcome some of the limitations of the DOCENTIA Program, adapting the development of teachers' professional competences to the organizational expectations expressed in the university's strategic lines. In order to do this, it defines the relevant personal and professional competences, actions and behaviors needed to fulfill the mission of the university, integrating missions and objectives in a system of professional and personal improvement accompanied by the academic director, rewarding excellence in strategic and organizational terms, and allowing delegation, autonomy and direction systems of teachers to adjust, redefine and adapt to university governance system.

Keywords: teaching performance assessment; teaching quality; university teaching skills; management by missions.

Sumario. 1. Introducción. 2. Objetivos de la investigación. 3. Evaluación de la actividad docente en la universidad. 4. Evaluación docente, apreciación del desempeño: competencias y misiones. 5. Metodología empleada en el diseño del modelo de desarrollo y evaluación de desempeño docente en la UFV. 6. Discusión del modelo. 7. Conclusiones. 8. Referencias bibliográficas.

Cómo citar: De Dios-Alija, T.; García Ramos, J.M. y Muñoz-Pérez, S. (2017). Diseño de un modelo de evaluación y desarrollo docente en una universidad privada. Revista Complutense de Educación, 28 (1), 61-80

\section{Introducción}

La definición más extendida de la dirección de una organización es conseguir resultados a través de otras personas, sin embargo la teoría de gerencia y gobernabilidad más aceptada en la actualidad es la que defiende un liderazgo situacional, en el que quien tiene la responsabilidad de dirigir lo hace en función del contexto, las exigencias técnicas propias y de las personas que colaboran para el logro de unos objetivos y el ambiente social, cultural y físico en el que se trabaja.

Fue McGregor (1960) el primero que investigó sobre el funcionamiento y análisis de resultados de los programas formativos dirigidos a la enseñanza de métodos de dirección en las organizaciones, llegando a la conclusión de que con demasiada frecuencia no lograban lo que pretendían. Sin embargo, su aportación fue clave para comprender el peso que tiene en la gestión empresarial, el concepto de hombre, de quien tiene la responsabilidad de la dirección de las personas. O bien se cree en un ser humano al que le disgusta el trabajo y si puede lo elude y por tanto es necesario "controlar" su actividad; o bien, se considera que la persona encuentra el trabajo como algo propio de la actividad humana y por tanto se puede confiar en su autocontrol, si existe un compromiso en cuanto a los objetivos adecuados y las recompensas derivadas de su logro. Es evidente que la actividad directiva difiere mucho, si se parte de una u otra convicción. 
Del primer enfoque surge una dirección basada en la organización, la planificación, la coordinación y el control, con patrones de liderazgo marcados por el poder y la rendición de cuentas. Del segundo, nace el interés por el estudio de la conducta humana, los valores, las creencias, la motivación en el entorno laboral y el funcionamiento de los grupos de trabajo. Es así como surgen nuevas teorías de dirección orientadas a la mejora de la conducta organizacional, la integración de las personas, la identificación del talento, la adecuación persona-puesto, el desarrollo personal y profesional, el cuidado por la comunicación y el clima institucional (García Ramos,2002: 105-126) y una mayor cooperación entre los integrantes de la estructura organizacional.

En este ámbito de estudio surge también la necesidad de conocer el modo en qué cada persona contribuye al logro de unos objetivos organizacionales. Así, se construyen algunas herramientas que tratan de ayudar a identificar cómo e incluso cuánto valor aporta cada persona en la realización de la actividad propia del puesto de trabajo que ocupa. Esta técnica se ha denominado "evaluación del desempeño y/o rendimiento".

En el ámbito empresarial la evaluación del desempeño se define como un procedimiento sistemático para medir, evaluar, e influir sobre los atributos, comportamientos y resultados relacionados con el trabajo (Dolan y otros, 2003).

La valoración del rendimiento implica identificar las dimensiones que afectan al éxito de la organización, medir según estándares la adecuación del desempeño del trabajador y gestionarlo, informando a cada uno sobre su rendimiento anterior y ayudándole a alcanzar un rendimiento superior (Gómez-Mejía, Balkin y Cardy, 2001).

La evaluación de resultados es un proceso destinado a determinar y comunicar a los empleados la forma en que están desempeñando su trabajo y en principio, elaborar planes de mejora (Byars y Rue, 1997).

Estos términos, evaluación del desempeño, valoración del rendimiento y evaluación de resultados, se emplean a menudo como sinónimos, sin embargo entre ellos existen diferencias. Mientras que el estudio del desempeño suele enfocarse al análisis de la actuación, es decir la apreciación y calificación del comportamiento en el trabajo (calidad); si se trata de valorar el rendimiento, lo que se contempla es la productividad, el resultado en relación con los medios o el esfuerzo invertido (performance) en términos de oportunidad (cantidad).

No obstante, el objetivo que pretende cualquiera de estos procesos técnicos y sistemáticos, es identificar y medir de manera continua el modo de trabajar y/o los resultados obtenidos:

- Evaluar competencias y oportunidades de mejora del profesorado.

- Detectar el grado de ajuste entre la persona y la misión del puesto docente que desempeña.

- Apreciar, valorar y reconocer los aspectos y resultados positivos del desempeño profesional.

- Orientar el desarrollo de áreas susceptibles de mejora en la actividad docente, investigadora y de gestión académica.

- Potenciar la comunicación ascendente, descendente y horizontal entre el claustro y la dirección académica. 
- Generar sentimiento de pertenencia a la organización universitaria.

- Validar criterios de selección de profesores.

- Detectar necesidades de formación y desarrollo.

- Actualizar los sistemas de reconocimiento y compensación del profesorado.

En el entorno de las instituciones públicas, incluidas las universidades, se ha definido la evaluación del desempeño como el procedimiento mediante el cual se mide y valora la conducta profesional y el rendimiento o logro de resultados. Esta evaluación es un proceso sistemático y periódico de estimación cuantitativa y cualitativa del grado de eficacia de una persona en su puesto de trabajo, es, por tanto, un procedimiento de valoración del rendimiento laboral individual y de su potencial laboral (Ley 7/2007, de 12 de abril, del Estatuto Básico del Empleado Público, artículo 20.1).

En el ámbito de la educación también comienza a apuntarse en esta dirección "en todos los países analizados, la evaluación del desempeño docente tiene como uno de sus propósitos básicos, formulados de manera más o menos explícita, la mejora de la calidad de la enseñanza; es decir, buscar colaborar con el desarrollo profesional del docente mediante el mantenimiento o la optimización del desempeño y los resultados de la enseñanza" (Murillo, González, Rizo, 2007).

\section{Objetivos de la investigación}

Al profesorado universitario se le viene evaluando desde hace décadas, tal como exponen los autores representados en la tabla 1 (García Ramos, 1997).

Los modelos de evaluación actuales, en general:

- Evalúan procesos de enseñanza-aprendizaje, pero no contribuyen a lograr los objetivos estratégicos de la universidad.

- Están muy enfocados a resultados y menos a la mejora continua del proceso de aprendizaje.

- No implica a los responsables de ejercer el liderazgo en la organización, por tanto no potencian el compromiso de los profesores con la Dirección Académica.

- Solo en ocasiones permiten identificar necesidades de formación y desarrollo.

- No siempre incentivan la calidad y la excelencia docente.

La pretensión de esta investigación es comprobar la posibilidad real de implantar en el ámbito universitario un nuevo sistema de apreciación del desempeño, que supere algunas de las limitaciones que los modelos de evaluación del profesorado mantienen en la actualidad. 


\begin{tabular}{|c|c|c|c|c|c|}
\hline $\begin{array}{c}\text { Dimensiones } \\
\text { comunes } \\
\text { Trent y Cohen } \\
1973\end{array}$ & $\begin{array}{c}\text { SEFQ } \\
\text { Marsh } \\
1982 \text { y } 1987\end{array}$ & $\begin{array}{c}\text { Endeavour } \\
\text { Frey, } \\
\text { Leonard y } \\
\text { Beaty } \\
1975\end{array}$ & $\begin{array}{c}\text { CEQ } \\
\text { Ramsden } \\
1991\end{array}$ & $\begin{array}{c}\text { E.F.P.A } \\
\text { Tejedor } \\
1993\end{array}$ & $\begin{array}{c}\text { CEDA-MOD } \\
\text { (García Ramos } \\
1997\end{array}$ \\
\hline $\begin{array}{l}\text { 1.Estructura y } \\
\text { claridad en la } \\
\text { explicación } \\
\text { 2. Positiva } \\
\text { actitud hacia la } \\
\text { discusión en } \\
\text { clase y la } \\
\text { exposición en } \\
\text { puntos } \\
\text { diferentes } \\
\text { 3. Estímulo del } \\
\text { interés, } \\
\text { motivación y } \\
\text { reflexión del } \\
\text { alumno } \\
\text { 4. Prestar } \\
\text { atención } \\
\text { individual al } \\
\text { alumno } \\
\text { 5.Entusiasmo }\end{array}$ & $\begin{array}{l}\text { 1. Aprendizaje- } \\
\text { Valor } \\
\text { 2. Interacción } \\
\text { con el grupo } \\
\text { 3. Raport } \\
\text { individual } \\
\text { 4. Exámenes- } \\
\text { calificaciones } \\
\text { 5. Carga de } \\
\text { trabajo- } \\
\text { dificultad } \\
\text { 6.Organización- } \\
\text { claridad } \\
\text { 7.Entusiasmo } \\
\text { 8.Amplitud de } \\
\text { enfoque } \\
\text { 9.Trabajo } \\
\text { extraclase- } \\
\text { lecturas }\end{array}$ & $\begin{array}{l}\text { 1. Logros de } \\
\text { los alumnos } \\
\text { 2. Discusión } \\
\text { en clase } \\
\text { 3. Atención } \\
\text { personal } \\
\text { 4.Calificacione } \\
\text { s } \\
\text { 5. Carga de } \\
\text { trabajo } \\
\text { 6. Claridad de } \\
\text { presentación } \\
\text { 7.Oganización- } \\
\text { planificación }\end{array}$ & $\begin{array}{l}\text { 1. Enseñanza } \\
\text { adecuada } \\
\text { 2. Correcta } \\
\text { orientación sobre } \\
\text { la marcha del } \\
\text { aprendizaje del } \\
\text { alumno } \\
\text { 3. Claridad en los } \\
\text { objetivos del } \\
\text { programa propio } \\
\text { y en lo que se } \\
\text { espera de uno } \\
\text { 4. Adecuada } \\
\text { carga de trabajo } \\
\text { 5. Adecuada } \\
\text { evaluación- } \\
\text { calificación del } \\
\text { aprendizaje } \\
\text { 6. Estímulo y } \\
\text { motivación al } \\
\text { alumno en su } \\
\text { responsabilidad } \\
\text { (a los estudiantes } \\
\text { se les dan } \\
\text { muchas } \\
\text { opciones, en el } \\
\text { trabajo que } \\
\text { realizan) }\end{array}$ & $\begin{array}{l}\text { 1. } \\
\text { Cumplimiento } \\
\text { de obligaciones } \\
\text { 2. Calidad y } \\
\text { desarrollo del } \\
\text { programa } \\
\text { 3.Dominio de la } \\
\text { asignatura } \\
\text { 4.Interacción } \\
\text { con los alumnos } \\
\text { 5.Recursos } \\
\text { utilizados y } \\
\text { prácticas } \\
\text { 6.Exámenes }\end{array}$ & $\begin{array}{l}1 . \\
\text { Programación- } \\
\text { organización de } \\
\text { la enseñanza } \\
\text { 2.Dominio de } \\
\text { contenidos- } \\
\text { claridad } \\
\text { expositiva } \\
\text { 3.Motivación de } \\
\text { aprendizaje- } \\
\text { incremento de } \\
\text { interés del } \\
\text { alumno } \\
\text { 4. Interacción } \\
\text { con el grupo de } \\
\text { clase. } \\
\text { 5. Atención } \\
\text { individual al } \\
\text { alumno. } \\
\text { 6. Evaluación- } \\
\text { exámenes. }\end{array}$ \\
\hline
\end{tabular}

Tabla 1. Dimensiones implicadas en diferentes instrumentos de evaluación docente (por el alumno).

La hipótesis de partida es que el sistema propuesto permitirá:

- Adecuar el desarrollo de las competencias profesionales de los profesores a las expectativas organizacionales expresadas en las líneas estratégicas de la universidad.

- Definir las competencias, acciones y comportamientos profesionales y personales relevantes para el cumplimiento de la Misión universitaria y evaluar la contribución y adecuación de la realización de cada persona a los mismos.

- Integrar resultados y competencias en un sistema de mejora profesional y personal, acompañado por el director académico, que facilita el aprendizaje y exige formación continua.

- Recompensar la excelencia en términos estratégicos (resultados) y organizativos (competencias - misión). 
Permitir que los sistemas de delegación, de autonomía y de dirección de los profesores se ajusten, se redefinan y se adapten al sistema de gobierno de la universidad.

Por supuesto, todo ello apoyado en el Sistema de Evaluación Institucional, dentro del cual, el programa DOCENTIA es una pieza clave.

\section{Evaluación de la actividad docente en la universidad.}

Con el objetivo de satisfacer la necesidad del sistema educativo español, de garantizar la calidad del desempeño de la labor del profesorado universitario y favorecer su desarrollo y reconocimiento, la Agencia Nacional de Evaluación de la Calidad y Acreditación (ANECA) ha impulsado la implantación del Programa de Apoyo a la Evaluación de la Actividad Docente (DOCENTIA).

En la UFV se viene realizando la evaluación de la actividad del profesorado mediante un procedimiento sistemático y permanente de recogida, análisis, interpretación y valoración de información relevante acerca de la labor docente, que ayuda a comprender la realidad, invitando al docente a reflexionar sobre su actividad y a tomar las decisiones oportunas para su perfeccionamiento profesional y en consecuencia, para la mejora institucional (evaluación formativa).

Este modelo de evaluación implantado en 1994 y mejorado en los sucesivos años, (García Ramos, 1999:417-443) ha sustentado el programa DOCENTIA de la UFV, acreditado por ANECA y la Agencia de Calidad, Acreditación y Prospectiva de las Universidades de Madrid (ACAP) en 2013 (hoy Fundación Madri+d), que entronca especialmente con una de las líneas estratégicas de la Universidad: la excelencia académica.

El Programa Docentia de la UFV considera cuatro dimensiones como objeto de evaluación de la actividad docente: 1. Planificación de la docencia, 2. Desarrollo de la docencia, 3. Resultados y 4. Mejora e Innovación. La evaluación se plantea desde el análisis de dieciséis subdimensiones (representadas en la siguiente tabla), a través de las cuales se pretende conocer cómo se desarrolla la actividad docente, identificar las mejores prácticas de los profesores en relación con su actividad en el aula, medir los resultados y por último, identificar la contribución a la innovación y a la mejora docente. 

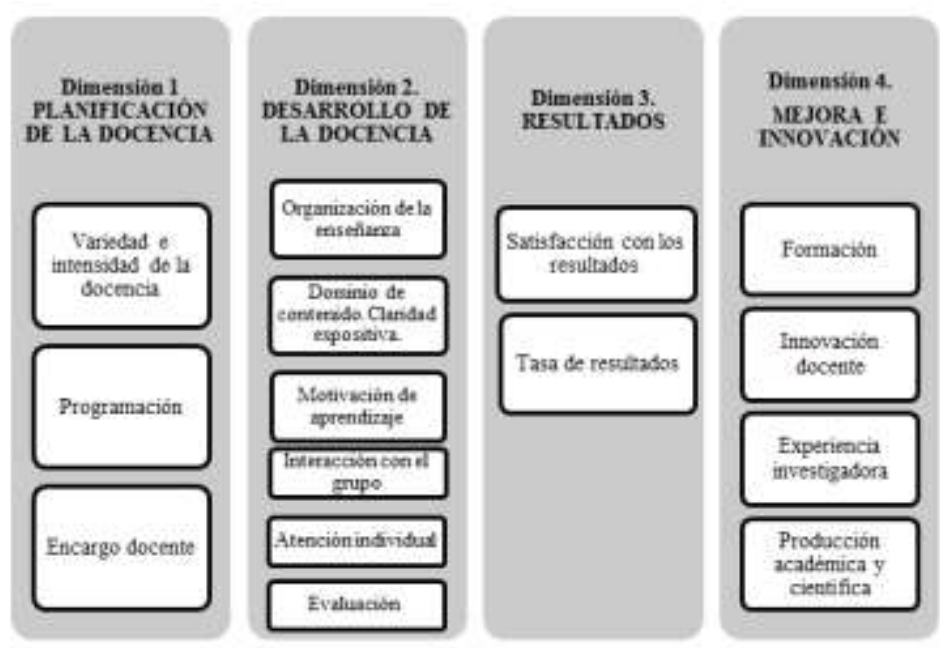

Tabla 2. Dimensiones y criterios a considerar en la evaluación

En el análisis por núcleos teóricos, en concreto en la dimensión Desarrollo de la docencia y Resultados, en la que se tiene en cuenta sobre todo la percepción de los estudiantes, se muestra (tal como se puede observar en la tabla 2 y el gráfico 1) que en 2013-2014, la valoración es muy satisfactoria en todos los núcleos y satisfactoria en "Interacción con el grupo en clase". La tendencia desde el 2011 en la media (en una escala de 6 puntos) de evaluación docente de la UFV es creciente en "Dominio de contenidos. Claridad Expositiva", "Motivación de aprendizaje", "Interacción con el grupo de clase", "Atención individual al alumno" y "Satisfacción con los Resultados". También la media global aumenta en estos años.

El núcleo "Cumplimiento de obligaciones" se considerará en el Programa DOCENTIA una dimensión 0, es decir, un requisito básico en el ejercicio de todo docente.

\begin{tabular}{lccc}
\hline \multicolumn{1}{c}{ Núcleos Teóricos } & $\mathbf{2 0 1 1 - 2 0 1 2}$ & $\mathbf{2 0 1 2 - 2 0 1 3}$ & $\mathbf{2 0 1 3 - 2 0 1 4}$ \\
\hline 1- Programación-organización de la enseñanza. & 4,66 & 4,73 & 4,69 \\
\hline 2- Dominio de contenidos. Claridad expositiva. & 4,72 & 4,74 & 4,76 \\
\hline 3- Motivación de aprendizaje. & 4,19 & 4,35 & 4,55 \\
\hline 4- Interacción con el grupo de clase. & 4,32 & 4,46 & 4,49 \\
\hline 5- Atención individual al alumno. & 4,60 & 4,68 & 4,71 \\
\hline 6- Evaluación. & 4,51 & 4,61 & 4,52 \\
\hline 7- Resultados. & 4,32 & 4,44 & 4,54 \\
\hline 8- Cumplimiento de Obligaciones & 4,83 & 4,88 & 4,91 \\
\hline MEDIA GLOBAL & 4,52 & 4,61 & 4,65 \\
\hline
\end{tabular}

Tabla 3: Notas medias por núcleos teóricos de la UFV en evaluación docente. Comparativa 2011-2014. 


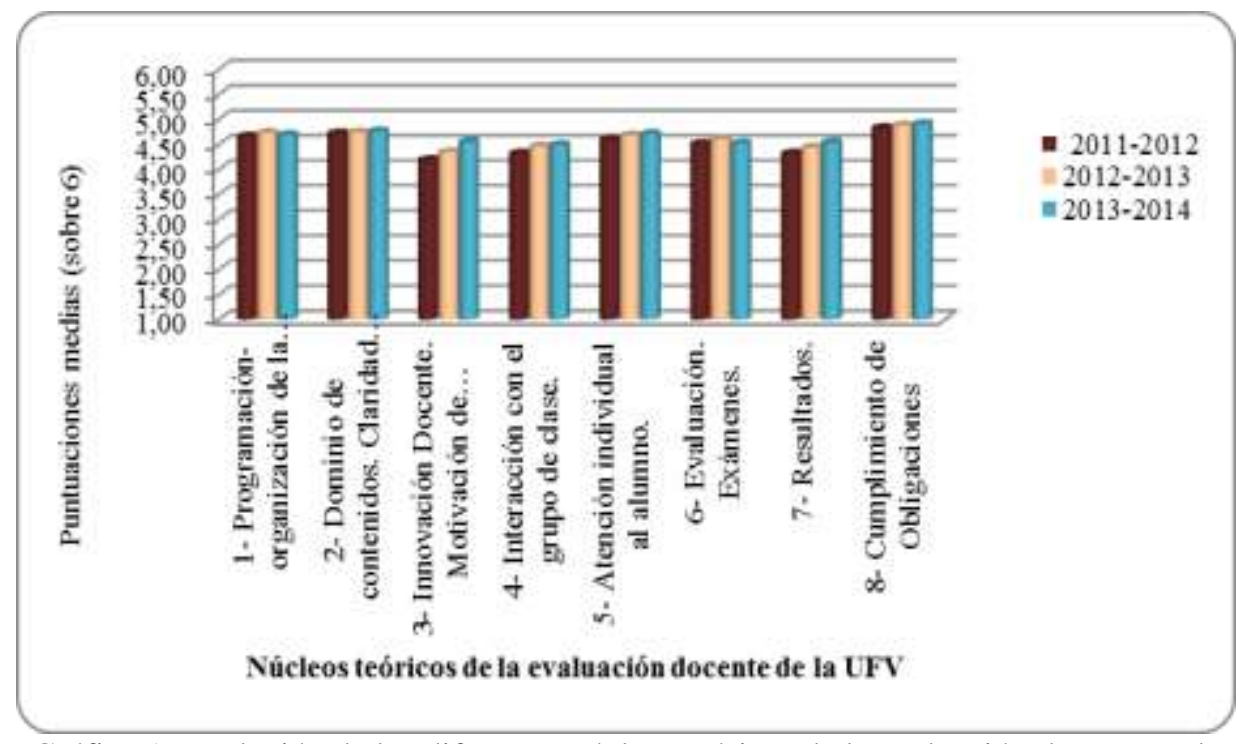

Gráfico 1: Evolución de los diferentes núcleos teóricos de la evaluación docente en la UFV y evolución global de la UFV 20011-2014

Respecto a las valoraciones globales por Titulación de Grado, el 100\% de las titulaciones mejoran o mantienen estables sus valoraciones de la satisfacción del alumnado La media global de la universidad refleja un nivel de satisfacción del alumnado muy elevado ( 7,8 sobre 10 ó 4,65 sobre 6).

\begin{tabular}{|c|c|c|c|c|c|c|c|c|c|}
\hline GRADOS & $\begin{array}{c}\begin{array}{c}\text { Media } \\
\text { (sobre 6) }\end{array} \\
\text { 2011- } \\
2012 \\
\end{array}$ & $\begin{array}{c}\text { Valor } \\
\text { sobre } \\
10 \text { puntos }\end{array}$ & $\begin{array}{c}\text { Media } \\
\text { (sobre 6) } \\
\text { 2012- } \\
2013 \\
\end{array}$ & $\begin{array}{l}\text { Valor } \\
\text { sobre 10 } \\
\text { puntos }\end{array}$ & $\begin{array}{c}\text { Varia- } \\
\text { ción } \\
\% \\
\text { anual } \\
11 / 12- \\
12 / 13 \\
\end{array}$ & $\begin{array}{c}\text { Media } \\
\text { (sobre 6) } \\
2013- \\
2014\end{array}$ & $\begin{array}{l}\text { Valor } \\
\text { sobre } 10 \\
\text { puntos }\end{array}$ & $\begin{array}{c}\text { Varia- } \\
\text { ción \% } \\
\text { anual } \\
12 / 13- \\
13 / 14 \\
\end{array}$ & $\begin{array}{l}\text { * Evolución } \\
\text { Cualitativa }\end{array}$ \\
\hline Bella Artes & 4,62 & $\begin{array}{c}\text { NOTABLE } \\
8 \\
\end{array}$ & 4,84 & $\begin{array}{c}\text { NOTABLE } \\
8.5 \\
\end{array}$ & $4,76 \%$ & 4,69 & $\begin{array}{c}\text { NOTABLE } \\
8 \\
\end{array}$ & $-3,10 \%$ & ESTABLE \\
\hline Diseño & 4,25 & $\begin{array}{c}\text { NOTABLE } \\
7 \\
\end{array}$ & 4,39 & $\begin{array}{c}\text { NOTABLE } \\
7 \\
\end{array}$ & $3,19 \%$ & 4,31 & $\begin{array}{c}\text { NOTABLE } \\
7 \\
\end{array}$ & $-1,82 \%$ & ESTABLE \\
\hline Medicina & 4,32 & $\begin{array}{c}\text { NOTABLE } \\
7 \\
\end{array}$ & 4,51 & $\begin{array}{c}\text { NOTABLE } \\
8 \\
\end{array}$ & $4,47 \%$ & 4,87 & $\begin{array}{c}\text { NOTABLE } \\
8 \\
\end{array}$ & $7,98 \%$ & MEJORA \\
\hline $\mathrm{ADE}$ & 4,51 & $\begin{array}{c}\text { NOTABLE } \\
8 \\
\end{array}$ & 4,62 & $\begin{array}{c}\text { NOTABLE } \\
8 \\
\end{array}$ & $2,49 \%$ & 4,52 & $\begin{array}{c}\text { NOTABLE } \\
7,5 \\
\end{array}$ & $-2,16 \%$ & ESTABLE \\
\hline Biotecnolog. & 4,41 & $\begin{array}{c}\text { NOTABLE } \\
7.5 \\
\end{array}$ & 4,57 & $\begin{array}{c}\text { NOTABLE } \\
8 \\
\end{array}$ & $3,60 \%$ & 4,60 & $\begin{array}{c}\text { NOTABLE } \\
8 \\
\end{array}$ & $0,66 \%$ & ESTABLE \\
\hline $\begin{array}{l}\text { Comunica. } \\
\text { Audiovisual }\end{array}$ & 4,44 & $\begin{array}{c}\text { NOTABLE } \\
7.5\end{array}$ & 4,47 & $\begin{array}{c}\text { NOTABLE } \\
7.5\end{array}$ & $0,68 \%$ & 4,49 & $\begin{array}{c}\text { NOTABLE } \\
7,5\end{array}$ & $0,45 \%$ & ESTABLE \\
\hline Derecho & 4,76 & $\begin{array}{c}\text { NOTABLE } \\
8\end{array}$ & 4,34 & $\begin{array}{c}\text { NOTABLE } \\
7\end{array}$ & $\begin{array}{c}- \\
8,88 \%\end{array}$ & 4,72 & $\begin{array}{c}\text { NOTABLE } \\
8\end{array}$ & $8,76 \%$ & MEJORA \\
\hline Excellens & 4,64 & $\begin{array}{c}\text { NOTABLE } \\
8 \\
\end{array}$ & 4,59 & $\begin{array}{c}\text { NOTABLE } \\
8 \\
\end{array}$ & $\begin{array}{c}- \\
1,05 \% \\
\end{array}$ & 4,58 & $\begin{array}{c}\text { NOTABLE } \\
8 \\
\end{array}$ & $-0,22 \%$ & ESTABLE \\
\hline Ing. Inform. & 4,7 & $\begin{array}{c}\text { NOTABLE } \\
8 \\
\end{array}$ & 4,53 & $\begin{array}{c}\text { NOTABLE } \\
8 \\
\end{array}$ & $\begin{array}{c}- \\
3,68 \% \\
\end{array}$ & 4,47 & $\begin{array}{c}\text { NOTABLE } \\
7,5 \\
\end{array}$ & $-1,32 \%$ & BAJA \\
\hline Periodismo & 4,38 & $\begin{array}{c}\text { NOTABLE } \\
7 \\
\end{array}$ & 4,65 & $\begin{array}{c}\text { NOTABLE } \\
8 \\
\end{array}$ & $6,06 \%$ & 4,48 & $\begin{array}{c}\text { NOTABLE } \\
7,5 \\
\end{array}$ & $-3,66 \%$ & ESTABLE \\
\hline Publicidad & 4,67 & $\begin{array}{c}\text { NOTABLE } \\
8 \\
\end{array}$ & 4,47 & $\begin{array}{c}\text { NOTABLE } \\
7.5 \\
\end{array}$ & $4, \overline{24 \%}$ & 4,59 & $\begin{array}{c}\text { NOTABLE } \\
8 \\
\end{array}$ & $2,68 \%$ & MEJORA \\
\hline
\end{tabular}




\begin{tabular}{|c|c|c|c|c|c|c|c|c|c|}
\hline Arquitectura & 4,48 & $\begin{array}{c}\text { NOTABLE } \\
7.5\end{array}$ & 4,77 & $\begin{array}{c}\text { NOTABLE } \\
8\end{array}$ & $6,56 \%$ & 4,78 & $\begin{array}{c}\text { NOTABLE } \\
8\end{array}$ & $0,21 \%$ & ESTABLE \\
\hline CAFYD & 4,79 & $\begin{array}{c}\text { NOTABLE } \\
8 \\
\end{array}$ & 4,56 & $\begin{array}{c}\text { NOTABLE } \\
8 \\
\end{array}$ & $\begin{array}{c}- \\
4,80 \% \\
\end{array}$ & 4,49 & $\begin{array}{c}\text { NOTABLE } \\
7,5 \\
\end{array}$ & $-1,54 \%$ & BAJA POCO \\
\hline $\begin{array}{l}\text { Educación } \\
\text { infantil }\end{array}$ & 4,93 & $\begin{array}{c}\text { NOTABLE } \\
8.5 \\
\end{array}$ & 5,11 & $\begin{array}{c}\text { EXCELEN } \\
\text { TE } 9 \\
\end{array}$ & $3,65 \%$ & 4,85 & $\begin{array}{c}\text { NOTABLE } \\
8 \\
\end{array}$ & $-5,09 \%$ & ESTABLE \\
\hline $\begin{array}{l}\text { Educación } \\
\text { primaria }\end{array}$ & 4,93 & $\begin{array}{c}\text { NOTABLE } \\
8.5\end{array}$ & 4,95 & $\begin{array}{c}\text { NOTABLE } \\
8.5\end{array}$ & $0,36 \%$ & 4,84 & $\begin{array}{c}\text { NOTABLE } \\
8\end{array}$ & $-2,22 \%$ & ESTABLE \\
\hline Enfermería & 4,57 & $\begin{array}{c}\text { NOTABLE } \\
8\end{array}$ & 4,73 & $\begin{array}{c}\text { NOTABLE } \\
8\end{array}$ & $3,41 \%$ & 4,83 & $\begin{array}{c}\text { NOTABLE } \\
8\end{array}$ & $2,11 \%$ & MEJORA \\
\hline Fisioterapia & 4,58 & $\begin{array}{c}\text { NOTABLE } \\
8\end{array}$ & 4,87 & $\begin{array}{c}\text { NOTABLE } \\
8.5\end{array}$ & $6,25 \%$ & 4,77 & $\begin{array}{c}\text { NOTABLE } \\
8\end{array}$ & $-2,05 \%$ & ESTABLE \\
\hline Marketing & 4,63 & $\begin{array}{c}\text { NOTABLE } \\
8\end{array}$ & 4,41 & $\begin{array}{c}\text { NOTABLE } \\
7\end{array}$ & $\begin{array}{c}- \\
4,67 \%\end{array}$ & 4,36 & $\begin{array}{c}\text { NOTABLE } \\
7\end{array}$ & $-1,13 \%$ & ESTABLE \\
\hline Farmacia & 4,7 & $\begin{array}{c}\text { NOTABLE } \\
8\end{array}$ & 4,71 & $\begin{array}{c}\text { NOTABLE } \\
8\end{array}$ & $0,27 \%$ & 4,78 & $\begin{array}{c}\text { NOTABLE } \\
8\end{array}$ & $1,49 \%$ & MEJORA \\
\hline Psicología & - & - & 4,84 & $\begin{array}{c}\text { NOTABLE } \\
8.5\end{array}$ & - & 4,67 & $\begin{array}{c}\text { NOTABLE } \\
8\end{array}$ & $-3,51 \%$ & BAJA \\
\hline $\begin{array}{l}\text { UFV- } \\
\text { Global }\end{array}$ & 4,52 & $\begin{array}{c}\text { NOTABLE } \\
8\end{array}$ & 4,61 & $\begin{array}{c}\text { NOTABLE } \\
8\end{array}$ & $1,99 \%$ & 4,65 & $\begin{array}{c}\text { NOTABLE } \\
8\end{array}$ & $0,87 \%$ & ESTABLE+ \\
\hline
\end{tabular}

Tabla 4. Medias globales por titulaciones y media global UFV en evaluación docente. Cursos 2011-2012, 2012-2013 y 2013-2014

Para poner de manifiesto la progresión del nivel de satisfacción de los alumnos por sus carreras, en la siguiente tabla se presenta la comparativa de las puntuaciones medias en la evaluación docente por titulación durante el periodo comprendido entre 2011 y 2014.

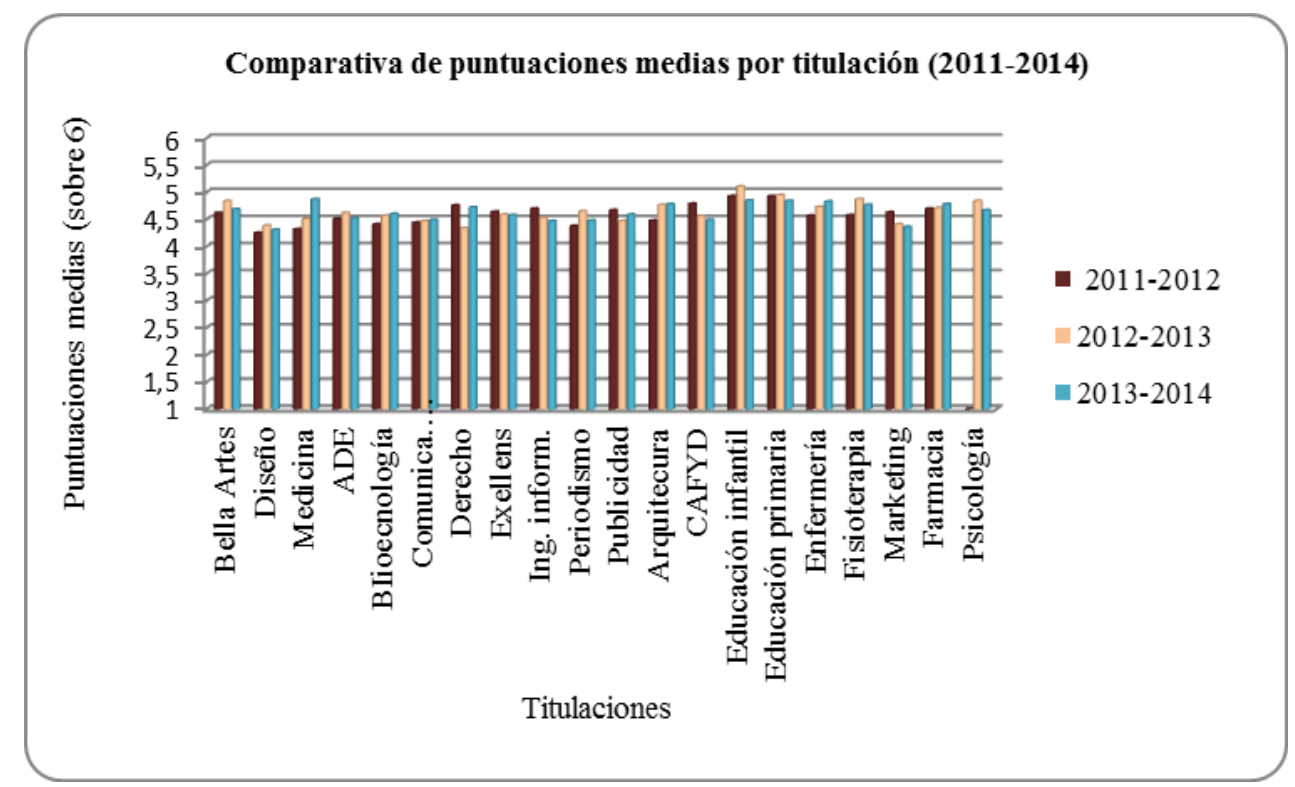

Gráfico 2: Evolución de las diferentes medias globales de la evaluación docente en la UFV y en las diferentes titulaciones 2011-2014 


\section{Evaluación docente, apreciación del desempeño: competencias y misiones}

Implantado el programa DOCENTIA, en la UFV se ha dado un paso más, implementando un sistema de evaluación del desempeño inspirado en la Dirección por Misiones (Cardona y Rey, 2006), que no sólo permita la mejora continua del profesor universitario, sino que además proporcione a los responsables académicos una pauta sistemática para ejercer la dirección y el desarrollo de los docentes e investigadores universitarios.

El profesor universitario, no realiza solo una función docente, además debe desarrollar una productiva actividad investigadora y en muchos casos una función de gestión que no podemos obviar en la evaluación, si intentamos considerar el desempeño completo de su actividad.

En la Dirección por Misiones se analiza lo que se ha dado en llamar Evaluación Integral, centrada en la contribución a la Misión global de la organización. "Este método combina el cumplimiento de los objetivos de la misión con otros aspectos cualitativos o de carácter intangible, como pueden ser factores ligados al comportamiento o al desarrollo personal de competencias" (Cardona y Rey, 2006).

No es suficiente con evaluar la docencia, analizar el proceso de planificación, desarrollo y evaluación del aprendizaje o la transferencia de conocimiento científico, además es necesario comprobar la contribución del profesor al cumplimiento de la Misión de la universidad en un periodo determinado. Por tanto, es importante considerar el punto de partida y posible perfeccionamiento de las competencias del profesor, que participa en los procesos universitarios no sólo como agente de la enseñanza, sino también como integrante de una comunidad de aprendizaje en desarrollo.

Analizar la docencia implica tomar en consideración no sólo la preparación, el desarrollo de la intervención didáctica directa y las acciones posteriores a ella, sino que además deben considerarse aspectos que trascienden lo experiencial y visible, que se derivan de las actitudes, interpretaciones de cada uno de los participantes que intervienen en el proceso de aprendizaje (Zabalza, 2003).

En el ámbito universitario y a la luz de las múltiples definiciones existentes sobre competencias, se concretan las competencias como la posibilidad real que tiene la persona de integrar:

- Datos, información, conceptos y conocimientos (saber/conocer).

- Habilidades, destrezas, capacidades, aptitudes (saber hacer/poder).

- Creencias, valores, motivaciones, actitudes, modos de actuación, hábitos y responsabilidades (saber ser/querer), para lograr tomar decisiones y actuar en contextos cotidianos reales y complejos.

Esta definición parte de la premisa de que la persona es un ser en desarrollo, dotado de inteligencia, voluntad, sensibilidad y conciencia de sí, que está constituido por diferentes dimensiones integradas (biológica, cognitiva, afectiva, social, espiritual) y conformado por aspectos físicos y psíquicos que la hacen única, singular e irrepetible y que se desarrolla durante la vida en tendencia a la plenitud. 
Por competencia entendemos la posibilidad de poner en práctica de forma integrada aquellos conocimientos adquiridos, aptitudes y rasgos de personalidad que permiten resolver situaciones diversas. El concepto de competencia va más allá de "saber" y de "saber hacer", incluye también incluye "saber ser" y "saber estar ".Esto requiere la comprensión, reflexión y discernimiento sobre la propia experiencia, que cada persona puede realizar por sí misma o con la ayuda de otros (seguimiento y feedback), de manera que pueda ponerse en práctica el aprendizaje adquirido teniendo en cuenta la responsabilidad personal en cada una de las acciones.

\section{Metodología empleada en el diseño del modelo de desarrollo y evaluación de desempeño docente en la UFV}

Algunas de las referencias relacionadas consultadas en las bases bibliográficas nacionales e internacionales, tratan sobre los elementos clave para la evaluación del desempeño de los docentes. (Elizalde Lora; Reyes Chávez, 2008), la evaluación del desempeño y carrera profesional docente (Murillo, González y Rizo, 2007), la evaluación del desempeño docente en la universidad (Rueda Beltrán, 2008), la formación de profesores basada en competencias (Gairín Sallan, 2011), la evaluación del desempeño docente (Pimentel Paredes, 1996), la evaluación del diseño de las 'Guías FORCOM de autoevaluación de competencias docentes' para el Espacio Europeo de Educación Superior (Álvarez Rojo, García García, Asensio Muñoz, Clares López y col., 2009), las competencias del docente universitario: la percepción del alumno, de los expertos y del propio protagonista (Mas Torelló, 2012), la planificación de evaluación de competencias en Educación Superior (García Sanz, Morillas Pedreño, 2011) y las características de la enseñanza universitaria desde las actuaciones profesionales del docente universitario (Rumbo Arcas, 1998).

Sin embargo no ha sido posible encontrar un modelo de evaluación de desempeño por misiones y competencias personales y profesionales, que se adecúe a las características particulares del entorno universitario. Por esta razón, desde el Vicerrectorado de Profesorado e Investigación y las áreas de Recursos Humanos, Desarrollo y Formación e Innovación Docente de la UFV se propone el diseño y discusión de un modelo, que partiendo del Programa DOCENTIA integre la definición y el compromiso con objetivos específicos de docencia, investigación y gestión ligados a competencias profesionales y personales, en aras a cumplir la misión universitaria.

La propuesta se configura partiendo de las evaluaciones de los alumnos en el aula, de la evaluación del director y de la autoevaluación del profesor (DOCENTIA). Se propone ampliar este punto de partida, identificando indicadores clave ligados a objetivos, misiones y actividades vinculadas.

Además, se añade una rúbrica para la valoración del perfil del profesor, a través de la apreciación de comportamientos observables asociados a un perfil de competencias. 
1. Planificación y orientación a resultados

2. Orientación al aprendizaje y desarrollo de las personas

3. Comunicación y transferencia del conocimiento

4. Trabajo cooperativo y en red (comunidad de aprendizaje)

5. Orientación a la mejora personal y profesional. Afán de superación

6. Innovación y gestión del cambio

7. Identificación y compromiso con la Misión.

Este perfil se determina a través del trabajo de consultoría realizado, durante el curso 2013-2014, en colaboración con profesionales externos a la universidad, expertos en la identificación, descripción y evaluación de perfiles competenciales asociados a la Misión organizacional.

Para proceder a la valoración de estas competencias se proporciona una herramienta a través de la que pueden identificarse y evaluarse una serie de comportamientos observables esenciales para el logro de la excelencia académica del profesorado. Cada competencia es definida y descrita a través de dimensiones que se recogen y gradúan en rúbricas, que son sometidas a revisión y juicio por diferentes agentes de la universidad (Dirección de Recursos Humanos, Dirección de la Oficina de Transferencia de Resultados de Investigación, Directores académicos, profesores, Personal de Administración y Servicios, asesores externos...).

Una vez establecidos los puntos de partida (DOCENTIA y perfil de competencias del profesorado) para la evaluación e identificación de áreas de mejora, se propone un sistema que permita realizar la propuesta de un plan de desarrollo personal y profesional.

Por tanto el modelo propuesto para el desarrollo y evaluación del desempeño docente se perfila como un procedimiento sistemático de apreciación de puntos fuertes en la actuación de cada profesor, identificación de áreas de mejora, priorización de metas y propuesta de un plan de acción personal que apunte a la mejora profesional y al cumplimiento de objetivos institucionales.

Este nuevo sistema de evaluación propuesto en la UFV, conocido como Sistema de Desarrollo y Evaluación del Desempeño Docente (SD3),permite identificar, orientar, seguir, revisar y mejorar el desempeño de las personas para que estas logren un mejor desarrollo de su labor docente, investigadora y de gestión de manera continuada.

\subsection{Descripción del Sistema de desarrollo y evaluación del desempeño docente (SD3)}

El sistema de desarrollo docente y evaluación del desempeño (SD3) es un sistema de dirección y seguimiento personalizado cuyo objetivo es incrementar la eficacia de la organización mediante el conocimiento y mejora del desempeño de las personas, el desarrollo profesional de los profesores y la orientación coordinada de los equipos docentes hacia los objetivos generales.

El director académico, responsable de hacer efectiva la Misión a través de la planificación estratégica y desarrollo de planes de acción en cada Grado: Medicina, 
Derecho, Administración de empresas, Arquitectura, etc, deberá realizar una serie de entrevistas de evaluación/feedback con cada profesor de su claustro, para ayudar a aproximar los objetivos y acciones de mejora y evaluar los resultados obtenidos al final del proceso en cada periodo de desarrollo.

La entrevista de evaluación es un instrumento que permite, al director y al profesor, identificar tanto los objetivos de desempeño como realizar el seguimiento de los mismos de manera continuada. Sus objetivos son:

- Identificar el desempeño profesional.

- Mejorar de manera continuada la actuación de las personas en su puesto de trabajo.

- Orientar la labor docente a la excelencia académica.

- Conseguir el diálogo y la mutua comprensión entre el director académico y el profesor en cuanto a lo que se espera de éste, la forma en que satisface esa expectativa y lo que puede hacer para mejorar sus resultados como docente.

En esta entrevista director y profesor analizan de manera conjunta la actividad docente, investigadora y de gestión, para identificar tanto aquellos aspectos en los que la contribución de la persona destaca, como los que son susceptibles de mejora, con el objeto de proponer acciones que puedan ayudarle a emprender el camino de desarrollo profesional que necesita para alinearse con la estrategia de la titulación y de la universidad.

Las entrevistas de evaluación y feedback en la UFV se completan al menos, en tres momentos:

\section{Entrevista inicial}

Se realiza al inicio de curso académico para analizar las valoraciones en el último programa DOCENTIA y la apreciación de comportamiento observables ligados a las competencias del profesor UFV.

El director académico repasa con cada profesor los resultados de las evaluaciones, para poder realizar una estimación de posibles áreas de mejora y establece objetivos de desarrollo prioritarios.

Después y a través de una serie de preguntas interpelantes orientativas, director y profesor identifican la manera en que el profesor actúa en su labor como docente, investigador y/o gestor, evaluando el punto en el que actualmente se encuentra en cuanto al desarrollo de cada competencia. Para ello ambos utilizan las rúbricas (dimensionadas y graduadas) de evaluación, que sirven de guía para realizar la apreciación de comportamientos observables, de manera que pueda hacerse explícito el planteamiento de objetivos de mejora y el avance en el proceso de perfeccionamiento docente.

La evaluación se realiza sobre siete competencias genéricas, facilitando el relato de evidencias comportamentales que permitan identificar la posibilidad de desarrollo de cada una. En la tabla 5 se enumeran y definen las competencias.

Después, director y profesor determinan los objetivos en función de las misiones de las que cada profesor es responsable y priorizan las áreas de desarrollo que deberán trabajarse (a través de programas de desarrollo y/o formación) a lo 
largo del año, así como las acciones concretas a realizar en plazos determinados. Para su registro se utiliza el documento representado en la tabla 6.

\section{COMPETENCIAS}

1. Planificación y orientación a resultados

2. Orientación al aprendizaje y desarrollo de las personas

3. Comunicación y transferencia del conocimiento

\section{Trabajo} cooperativo y en red (comunidad de aprendizaje)

5. Orientación a la mejora personal y profesional. Afán de superación

6. Innovación y gestión del cambio

7. Identificación y compromiso con la Misión.

\section{DESCRIPCIÓN}

Definición del plan de trabajo para el logro de los resultados propuestos (seguimiento de objetivos -metas e indicadores-) propios y/o de la institución. Desarrollo de estrategias eficaces para la consecución de los objetivos y el plan propuesto. Ejecución de las acciones definidas cumpliendo en tiempo y forma con los resultados esperados.

Atención personal y acompañamiento a otros (alumnos, compañeros, ...) en la definición e impulso de planes de acción. Realización de acciones encaminadas al desarrollo de las personas, la configuración de equipos docentes y la formación de una verdadera comunidad de aprendizaje orientada a la búsqueda de la verdad.

Claridad (capacidad de síntesis), sentido y fundamentación en sus comunicaciones (orales y escritas). Consideración y adaptación al destinario. Adecuación de los medios/espacios de difusión del conocimiento (docente, investigador y de gestión). Disponibilidad y aceptación de críticas y comentarios a sus comunicaciones. Seguimiento de las comunicaciones realizadas. Transferencia del conocimiento.

Comprensión real de la institución universitaria y de su funcionamiento. Orientación a la implementación de nuevas estrategias de trabajo que supongan generar valor añadido en la comunidad universitaria. Coordinación y transdisciplinariedad con otros agentes (docentes, investigadores, gestores). Fomento de alianzas intra e interinstitucionales

Acciones de formación continua. Motivación hacia la mejora y reflexión sobre su desarrollo profesional y personal. Aprovechamiento de las experiencias que contribuyan a la mejora profesional basada en una reflexión continua sobre su práctica y sobre los resultados esperados.

Actitud positiva, abierta, proactiva y flexible frente a los cambios, generando e impulsando soluciones y nuevas formas de hacer que contribuyan a alcanzar la excelencia en el entorno cambiante actual. Desarrollo de actividades novedosas que activen su práctica profesional.

Compromiso con los principios y valores de la misión, dentro y fuera de la misma, hasta el punto de inspirar y comprometer a otros. En su comportamiento muestra que es una persona coherente, íntegra y honesta. Espíritu y vocación de servicio, búsqueda de la verdad y orientación a la responsabilidad social y al bien común. 


\begin{tabular}{|l|l|l|l|l|}
\hline 1) Áreas de responsabilidad & Objetivo & Acción & Indicador & Fecha \\
\hline Docencia & & & & \\
\hline Investigación & & & & \\
\hline Gestión (misión y objetivos del puesto) & & & & \\
\hline 2) Desarrollo de competencias & & & & \\
\hline $\begin{array}{l}\text { 3) Identificación de necesidades de formación y } \\
\text { desarrollo. }\end{array}$ & \multicolumn{3}{|l}{} \\
\hline
\end{tabular}

Tabla 6. Documento de registro de los objetivos fijados en la entrevista.

\section{Entrevista de seguimiento.}

En la entrevista de seguimiento, que se realiza sobre la mitad del curso académico, director y profesor contrastan la información referente a la consecución de los objetivos planteados, procediendo así a su evaluación. En caso necesario pueden reformular alguno de los objetivos o proponer alguno nuevo. Realizan el balance de la contribución de los programas de desarrollo y plantean la posibilidad de nuevas acciones de formación.

\section{Entrevista de cierre. Al finalizar el curso académico}

En la entrevista de cierre se realiza la evaluación final del cumplimiento de objetivos y se plantean acciones de mejora para el siguiente curso académico. Se estima el grado de contribución de la formación y los programas de desarrollo al logro de los resultados esperados.

\section{Discusión del modelo}

Una vez perfilada la propuesta del modelo para el desarrollo docente y la evaluación del desempeño del profesorado en la UFV, se somete a validación a través de Grupo de Discusión, entendiendo que esta es una "técnica no directiva que tiene por finalidad la producción controlada de un discurso por parte de un grupo de sujetos que son reunidos, durante un espacio de tiempo limitado, a fin de debatir sobre determinado tópico propuesto por el investigador" (Gil-Flores, 1993).

El grupo de discusión se configura con 11 personas (6 profesores de diferentes áreas de conocimiento y 5 son cargos académicos) que se organizan en tres subgrupos que procederán al análisis, discusión y propuestas de mejora de cada uno de las cuestiones que se componen el modelo presentado. La selección de las personas trata de asegurar la heterogeneidad de los miembros de la discusión y, de este modo, contar con la riqueza de perspectivas provenientes de distintas áreas y modos de hacer y entender la realidad de la práctica docente. La secuencia de trabajo se concreta en cuatro reuniones con una periodicidad mensual, cada una de dos horas de duración celebradas en las instalaciones de la universidad entre abril y julio de 2013.

Estas reuniones son moderadas por un representante del grupo de investigación. Además se cuenta con el apoyo de otras tres personas procedentes del Personal de Administración y Servicios que registran los detalles de la discusión, las cuales levantan acta consensuada de los argumentos esgrimidos en cada reunión y de los 
acuerdos alcanzados. Tres asesores externos ejercen roles de observación, verifican y contrastan la veracidad de los acuerdos alcanzados.

En cada reunión se aborda el análisis y la reflexión de cada aspecto de la propuesta con objeto de cuestionar la utilidad global del sistema de desarrollo y evaluación del desempeño propuesto (SD3).

Las conclusiones de trabajo de los grupos de discusión sobre el SD3 son las siguientes:

1. Este sistema puede mejorar la dirección académica y el desempeño del profesor.

2. Este sistema de desarrollo y evaluación debe estar orientado al cumplimiento de la Misión de la universidad y de su modelo educativo.

3. Se pretende que el sistema permita una evaluación flexible y personalizada, no sujeta a perfiles cerrados. Esto se conseguirá mediante la realización de una entrevista personalizada en la que el Director Académico ajusta con el profesor el compromiso de su trayectoria docente, investigadora y de gestión.

4. Es necesario definir los procedimientos mediante los cuales se realizará la valoración del desempeño de los profesores fomentando la homogeneidad entre las diferentes titulaciones, pero respetando la libertad de poder adaptarse a las particularidades de cada titulación.

5. Este sistema ayudará a detectar fortalezas y debilidades de los profesores, permitiendo la planificación de planes personalizados de formación para el desarrollo profesional y personal del docente.

6. No se recomienda la introducción de indicadores de resultados económicos (retribución variable), al menos hasta tener consolidado el modelo.

Finalizado el proceso de discusión y mejora, el grupo de investigación propone el diseño definitivo al Comité de Dirección de la universidad, quién decide someter a prueba el sistema actualizado. Para ello se cuenta con la colaboración los directores y el claustro de Arquitectura y Administración y Dirección de Empresas. La prueba piloto se desarrolla con éxito durante el curso 2013-2014.

\subsection{Desarrollo de la prueba piloto}

A continuación se describen los hitos en el desarrollo de este proyecto piloto con las dos titulaciones implicadas (Administración y Dirección de Empresas y Arquitectura).

- Para hacer un seguimiento cercano de los progresos en la implementación de esta prueba piloto, a partir de octubre de 2013 se programan reuniones semanales con las dos Direcciones Académicas que participan en el proceso (en adelante DDAA).

- Las DDAA informan acerca de la previsión de profesores que van a participar en la evaluación.

- Se realiza un taller formativo con los directores sobre el desarrollo de las entrevistas y la evaluación de evidencias comportamentales mediante 
identificación de incidentes críticos, útil en la evaluación de las competencias de los docentes.

- Se propone una herramienta con soporte informático para sistematizar la información necesaria para realizar este proceso de evaluación.

- Desde Rectorado se convoca una reunión para presentar el nuevo Sistema de Dirección y Desarrollo Docente a los profesores de las DDAA que participan en el proceso.

- Se ejecuta la prueba piloto evaluando a un total de 20 profesores de las áreas de Arquitectura y Administración y Dirección de empresas y se estudia la percepción de los implicados en el proceso.

\subsection{Análisis de resultados de la prueba piloto}

Para el seguimiento y posterior análisis de los resultados se pide a los directores y profesores participantes (dos directores y una muestra de 20 profesores), que redacten sus impresiones sobre el desarrollo de la prueba piloto. La información es recogida a través de una entrevista que contiene las siguientes preguntas:

1. Valoración global del grado de contribución de este proceso a la mejora profesional del profesor.

2. Utilidad del modelo para la dirección académica.

3. ¿La implantación de este modelo (orientado a la mejora del desempeño docente) puede contribuir al aprendizaje y la formación de profesionales centrados en la persona?

4. Evaluación global del proceso de implantación del SD3. Indica lo mejor y lo mejorable

5. Consejos o recomendaciones para la implantación en otros grados.

Se incorporan a continuación, a modo de conclusión de este estudio, algunos de los testimonios de los participantes, que presentamos como muestra representativa del amplio número de comentarios realizados:

- El SD3 permite valorar diferentes aspectos de la labor del profesorado y de este modo hacer una evaluación integral y por lo tanto más justa.

- Este modo de evaluar ayuda a detectar las fortalezas y las debilidades de los profesores de modo que permitirá adecuar sus tareas a aquellas áreas en las que destaquen por su excelencia y a su vez, se podrán detectar los puntos débiles.

- El compromiso que se establece con la persona permite hacerle conocedor de los objetivos institucionales y del título, mejora la comunicación y la comprensión de la misión global.

- Mejora la relación del Director con el profesor -se le considera al profesor como parte viva de la comunidad.

- Los profesores han desarrollado una mayor confianza y han definido la necesidad de medios para desarrollar mejor su labor-aceptando y promoviendo las propuestas razonables realizadas por la dirección en docencia, investigación y gestión-. 
- La evaluación se configura como una ayuda eficaz para el desarrollo de la vocación del profesor.

- Mayor conocimiento de los intereses del profesorado, de sus inquietudes, de su personalidad, de sus fortalezas y debilidades.

- Aumenta los vínculos entre los docentes en el desarrollo de los objetivos y mejor orientación hacia el desarrollo.

- Es un excelente mecanismo para incentivar y potenciar la motivación y el compromiso del profesorado con las distintas acciones y estrategias de la Universidad.

- Personalmente me ha facilitado el canal de comunicación con la Universidad y con el devenir presente y futuro de la misma.

- Ha permitido realizar actividades que profesionalmente me han enriquecido en la mejora en mi actividad profesional y docente.

También se identifican algunas sugerencias de mejora en el proceso:

Si desde la dirección somos capaces de ayudar al profesor a definir su actividad descubriendo como acompaña al desarrollo de su vocación como persona y docente y colaboramos en su camino, al detectar acciones que nos permitan crecer en comunidad y nos abrimos y desarrollamos las entrevistas desde su desarrollo integral....entonces será probable que en su labor diaria interioricen estos aspectos y contribuyan al aprendizaje y la formación de profesionales centrados en la persona.

Tal como podemos comprobar en la revisión de resultados, el sistema propuesto permite adecuar el desarrollo de las competencias docentes al cumplimiento de la misión de la UFV, proporcionando la mejora profesional y personal, recompensando la excelencia y permitiendo un sistema eficaz de Dirección Académica.

\section{Conclusiones}

La responsabilidad de dirigir la universidad hacia la excelencia académica en un entorno cambiante, pasa por conocer y establecer sistemas de gerencia y gobernabilidad que contribuyan a la misión que la organización pretende, promoviendo que cada persona desempeñe la misión que mejor pueda realizar, con objeto de propiciar un clima organizacional adecuado en el que puedan hacerse realidad los objetivos estratégicos planteados.

La finalidad de la evaluación del desempeño del profesorado es identificar lo que ocurre en la universidad en el ámbito de la docencia, la investigación y la gestión. Se trata de proponer procesos técnicos y sistemáticos que permitan conocer y medir de manera continua, el modo en que los profesores desarrollan su labor profesional para identificar oportunidades de mejora, detectar el grado de ajuste entre la persona y la misión del puesto que desempeña, apreciar, valorar y reconocer los aspectos y resultados positivos de la actuación, obtener información directa de los responsables de las misiones, potenciar la comunicación ascendente, 
descendente y horizontal, generar sentimiento de pertenencia a la organización, validar criterios de selección de personas, detectar necesidades de formación y desarrollo y actualizar los sistemas de reconocimiento y compensación.

Para validar una propuesta de este tipo, en la UFV se ha implementado un sistema de desarrollo y evaluación del desempeño docente que tiene la pretensión de superar algunas de las limitaciones del Programa DOCENTIA, adecuando el desarrollo de las competencias profesionales de los profesores a las expectativas organizacionales expresadas en las líneas estratégicas de la universidad, definiendo las competencias, acciones y comportamientos profesionales y personales relevantes para el cumplimiento de la Misión universitaria, integrando las misiones y objetivos en un sistema de mejora profesional y personal acompañado por el director académico, recompensando la excelencia en términos estratégicos y organizativos y permitiendo que los sistemas de delegación, de autonomía y de dirección de los profesores se ajusten, se redefinan y se adapten al sistema de gobierno de la universidad.

Tras el éxito de la implantación en 2014 en dos titulaciones, se procede a la extensión del modelo en todas las Direcciones Académicas de Grado. Así, en la actualidad participa en el proceso de evaluación y desarrollo el $20 \%$ de los docentes de la UFV, en tendencia la previsión es que en el curso 2015-2016 participe todo el profesorado que ahora es evaluado de acuerdo al Programa DOCENTIA, es decir el $100 \%$ de los contratados con una antigüedad de tres años como docentes.

Para medir el impacto de este sistema de dirección y evaluación del desempeño en la mejora continua se procederá al diseño de un modelo de seguimiento que obtendrá sus frutos en 2016 y que continuará revisándose en el futuro para comprobar la eficacia real del modelo presentado.

\section{Referencias bibliográficas}

Álvarez Rojo, V; García García, M.; Asensio Muñoz, I.I; Clares López, J. y col. (2009) Evaluación del diseño de las 'Guías FORCOM de autoevaluación de competencias docentes' para el Espacio Europeo de Educación Superior (EEES), Revista de docencia universitaria, Santiago de Compostela, 7 (4): 12.

García Ramos, J.M. (2002) La calidad de las instituciones educativas y algunas de sus dimensiones básicas, Revista de educación, 329: 105-126

García Ramos, J.M. (1997) Valoración de la competencia docente del profesor universitario: una aproximación empírica, Revista complutense de educación, 8, 2, pp. 81-108

García Ramos, J.M. (1999) Análisis multirrasgo-multimétodo en la validación de instrumentos para la evaluación de la calidad docente en Instituciones Universitarias, Revista Española de Pedagogía, 57, 214, pp. 417-443

Byars, LL. Y Rue, B. (1997) Gestión de Recursos Humanos, McGraw-Hill.

Cardona, P. Y Rey. C, (2006) Dirección por misiones, primeras experiencias de éxito, Economistas, 24(109): 101-107.

Dolan, S.L., Valle Cabrera, R., Jackson, S. E, Schuler, R.S. (2003). La gestión de los recursos humanos. McGraw-Hill, Madrid, $2^{\circ}$ ed. 
Elizalde LORA, L., \& Reyes Chávez, R. (2008). Elementos clave para la evaluación del desempeño de los docentes. Revista electrónica de investigación educativa, 10(SPE).

España. Art. 20.1 ley 7/2007 del Estatuto Básico del Empleado Público. BOE núm. 13, de 15 de enero de 2015, páginas 3056 a 3058 (3 págs.).

Gairín Sallan, J. (2011). La formación de profesores basada en competencias, Bordón 63 (1): 93-108.

García Sanz, M.P.; Morillas Pedreño, L. R. (2011). La planificación de evaluación de competencias en Educación Superior, Revista electrónica interuniversitaria de formación del profesorado, Zaragoza, 36 (141) 1: 113-124.

Gil Flores, J. (1993). La metodología de investigación mediante grupos de discusión, Enseñanza \&Teaching, № 10-11, 199-214.

Gómez-Mejía, L. R., Balkin D. B. y Cardy, R. L. (2001).Dirección y gestión de Recursos Humanos, Pearson Educación, Madrid.

Mas Torelló, O. (2012). Las competencias del docente universitario: la percepción del alumno, de los expertos y del propio protagonista, Revista de docencia universitaria, Santiago de Compostela, 10 (2): 299-318

McGregor, D. (1960). The human side of enterprise, New York, Mc Graw-Hill

Murillo, F. J., González, A., \& Rizo, M. (2007). Evaluación del desempeño y carrera profesional docente. Una panorámica de América y Europa. Santiago de Chile: UNESCO.

Pimentel Paredes, F. (1996). Evaluación del desempeño docente: propuesta complementaria al sistema de evaluación de la Universidad Nacional Autónoma de México (UNAM), Universidad de Salamanca.

Rueda Beltrán, M. (2008). La evaluación del desempeño docente en la universidad. Revista electrónica de investigación educativa, 10(SPE), 1-15.

Rumbo Arcas, B. (1998). Características de la enseñanza universitaria desde las actuaciones profesionales del docente universitario, Innovación educativa. Santiago de Compostela, 8:109-121.

Zabalza, M. A. (2003).Competencias docentes del profesorado universitario. Calidad y desarrollo profesional. Narcea Madrid. 